17.1

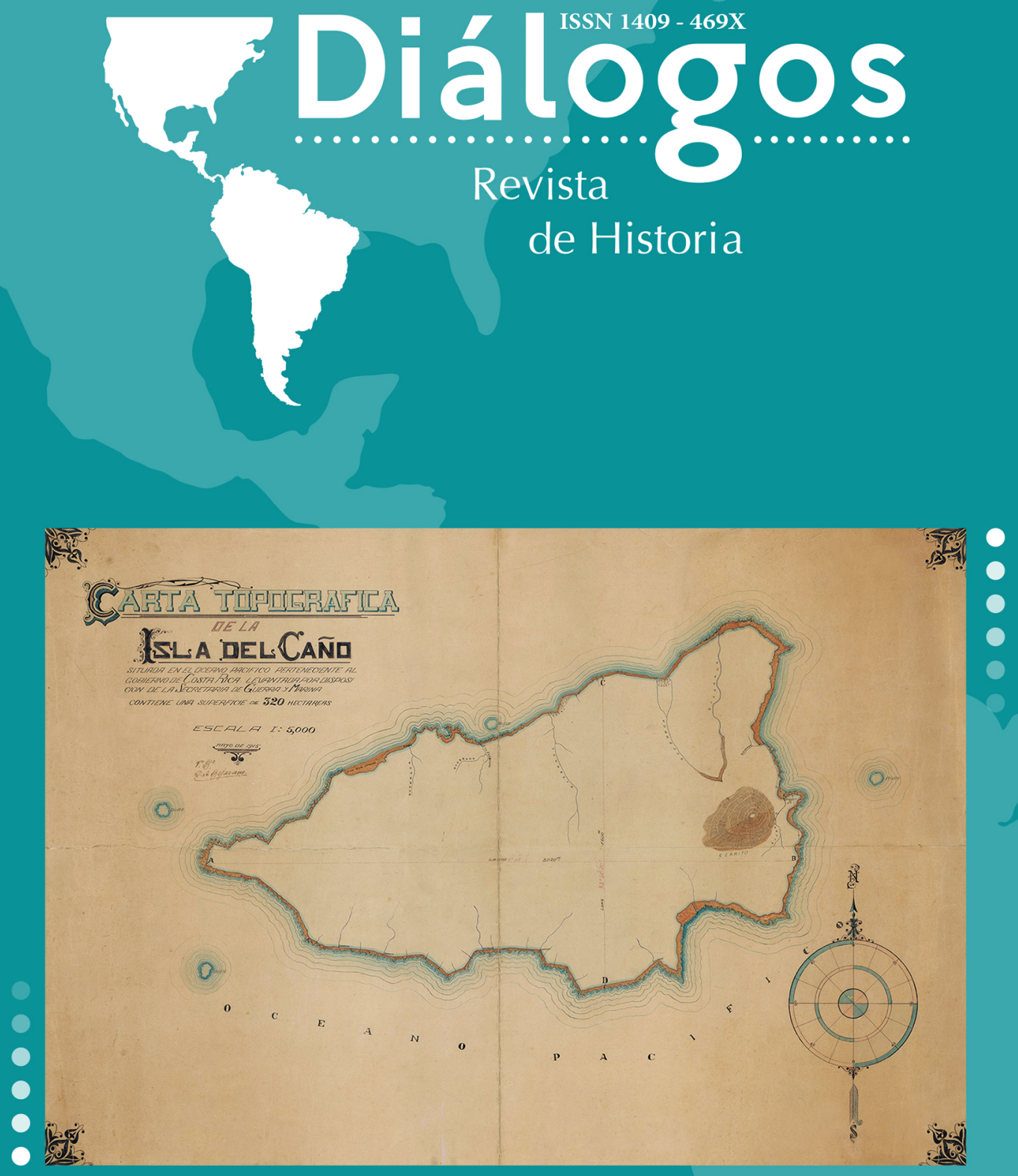

Escuela de Historia. Universidad de Costa Rica

Enero-junio 2016

url: http://revistas.ucr.ac.cr/index.php/dialogos/index 


\title{
LOS ESTUDIOS DE LA VIOLENCIA EN COLOMBIA ANTES DE LA VIOLENTOLOGÍA
}

Catalina Cartagena

\begin{abstract}
Resumen
Este artículo está estructurado analíticamente en dos partes. La primera despliega, en breve, un panorama general de la violencia en Colombia teniendo en cuenta los antecedentes y el desarrollo de la denominada violencia de mitad del siglo XX. La segunda parte revela un antes y un después de las interpretaciones sobre la violencia en relación con el proceso de institucionalización de las Ciencias Sociales en el país. En concreto, el objetivo de este artículo es mostrar cómo la institucionalización de las Ciencias Sociales repercutió sustancialmente en la construcción de un espacio académico dedicado especialmente al tema de la violencia, espacio que luego será reconocido como violentología.
\end{abstract}

Palabras claves: violencia, ciencias sociales, historia, Colombia.

\section{STUDIES OF VIOLENCE IN COLOMBIA BEFORE VIOLENTOLOGY}

\begin{abstract}
This article is analytically structured in two parts: The first, shortly presents an overview of violence in Colombia taking into account the history and development of the so-called violence during the first half of the twentieth century. The second part shows a before and after regarding the interpretations on violence in connection with the institutionalization of the social sciences in the country. Specifically, the aim of this article is to show how the institutionalization of the social sciences had a substantial impact on the construction of an academic space especially devoted to the subject of violence, which would then be recognized as violentology.
\end{abstract}

Keywords: violence, social sciences, history, Colombia.

Fecha de recepción: 2 de noviembre de 2014 • Fecha de aceptación: 27 de abril de 2015

- Catalina Cartagena - Investigadora y consultora independiente. Magister en Ciencias - Sociales de la Universidad Nacional General Sarmiento de Argentina y socióloga de la

- Universidad Nacional de Colombia. 


\section{INTRODUCCIÓN}

Este artículo está organizado en dos partes. La primera despliega, en breve, un panorama general de la violencia en Colombia haciendo énfasis en la contextualización de los antecedentes y desarrollo de la denominada violencia de mitad de siglo que fragmentó en dos la historia del país. La segunda parte revela un antes y un después de las interpretaciones sobre la violencia en relación con el proceso de institucionalización de las Ciencias Sociales en Colombia. Bajo esta estructura dedicamos especial atención a la producción de literatura sobre la violencia preliminar a la profesionalización de las disciplinas académicas, donde encontramos interpretaciones determinadas por afiliaciones afectivas e intenciones apologéticas o testimoniales. Y al mismo tiempo damos cuenta de la producción que emerge con un enfoque más científico y sistemático, tomando como punto de partida la obra cumbre de los años sesenta titulada: La Violencia en Colombia. El objetivo de este artículo es mostrar cómo la institucionalización de las Ciencias Sociales -en cuanto corpus de conocimientos, problemas y técnicas de investigación, procesado sistemáticamente y reconocido y aprobado por una comunidad epistémica (Palacios, 1994)- repercutió sustancialmente en la construcción de un espacio académico dedicado especialmente al tema de la violencia, espacio que luego será reconocido como violentología.

Aunque este artículo se centra en el periodo histórico particular transcurrido en la mitad del siglo XX y concebido por la historiografía colombiana e internacional como La Violencia, es pertinente anotar que la caracterización del conflicto armado en general es, aún hoy, fuente de debate prolongado y que al respecto no existe un consenso establecido (Pizarro, 2015, p. 43). Las nociones pasan por nombres como "conflicto social armado", "guerra civil" o "guerra contra la insurgencia", entre muchos. La diversidad de nominaciones tiene estrecha relación con las explicaciones de las causas sobre el fenómeno. Sin embargo, a pesar de esta multiplicidad y siguiendo la postura de Pizarro, la más común en la literatura especializada y en los documentos de la propia Mesa de Negociación de la Paz que se produce actualmente en La Habana, Cuba, entre el Estado colombiano y la guerrilla de las FARC, es la de "conflicto armado interno" (Pizarro, 2015, p. 45). Las características de este conflicto son las siguientes:

\footnotetext{
Se trata, en primer término, de un conflicto prolongado... ya sea que se inicie desde la Violencia (o antes), desde la emergencia de las guerrillas post-revolución cubana o a partir de los años ochenta del siglo pasado. En cualquiera de los tres casos, la confrontación armada en Colombia constituye una de las más antiguas del mundo. (Giraldo citado por Pizarro, 2015, pp. 33-34).
}

Otros elementos que caracterizan al conflicto colombiano actual son su carácter complejo, dada la gran cantidad de actores que participan, la multiplicidad de dimensiones que supone y factores que lo condicionan. Asimismo, se caracteriza por su discontinuidad y por las enormes diferencias regionales y geográficas en 
intensidad y magnitud. Finalmente, es necesario anotar que el conflicto en su fase actual se complejiza debido a la irrupción de los actores paramilitares, el tráfico de drogas y actividades ilícitas como el secuestro y la extorsión (Pizarro, 2015, p. 49).

Ahora bien, aunque el mote de violentología en el contexto colombiano se halla actualmente en desuso, el estudio sobre la violencia en general (la cual incluye en su interior la multiplicidad de caracterizaciones del fenómeno) en el campo académico de las Ciencias Sociales colombianas se ha consolidado y la temática es considerada fundamental dentro de los quehaceres propios de las disciplinas sociales. De igual manera, los intelectuales y expertos en la materia se han vinculado al Estado en distintas oportunidades a través de la participación de las comisiones académicas, las cuales, por medio de sus informes, tienen como meta contribuir en el entendimiento del conflicto armado y, en esta última etapa, colaborar con el buen término de las negociaciones de paz que actualmente se llevan a cabo en La Habana.

\section{EL PANORAMA GENERAL DE LA VIOLENCIA EN COLOMBIA}

Para empezar, es importante resaltar que dentro del panorama regional, el caso colombiano cuenta con la particularidad de tener la insurrección armada más extendida de América Latina, pues "sus orígenes datan de los comienzos de los años sesenta, de la época inmediatamente posterior a la revolución cubana y de las ofensivas nacionales contra las llamadas «repúblicas independientes»" (Chernick, 1996, p. 4). No obstante, si bien las guerrillas armadas se conformaron en la década de los sesenta, los conflictos sociales internos tienen antecedentes que aparecen desde comienzos de siglo. En este marco, la primera parte de este artículo contextualiza de manera general esos antecedentes que permitirán el desarrollo de la violencia de mitad de siglo y el posterior surgimiento de los grupos guerrilleros.

Siguiendo de cerca la postura del historiador colombiano Gonzalo Sánchez, ${ }^{1}$ el proceso conflictivo y paradigmático que ha desafiado Colombia, desde su emancipación del imperio español hasta la actualidad, denota el complejo devenir de la historia social y política republicana. El siglo XIX dejó 14 años de guerra independentista, 14 guerras civiles locales y dos guerras internacionales con Ecuador. El siglo XX, numerosos levantamientos locales, una guerra con Perú y la más importante insurrección popular en 1948 que dio lugar a una cruzada más larga: el acontecimiento que hoy todavía se conoce y describe con el término de La Violencia (Sánchez, 2007, p. 17), seguida después por la lucha contra las guerrillas, contra el narcotráfico y contra las fuerzas paramilitares.

En efecto, visto de ese modo, el conflicto social armado en Colombia pareciera presentarse como un acontecimiento permanente. Aun así este no goza de inmutabilidad, por el contrario, la variabilidad de los contextos de producción de las contiendas, de los actores protagonistas y de las motivaciones, lo particularizan dentro del ámbito latinoamericano y mundial. De esta manera partimos de la idea de 
que en Colombia para cada época hay relaciones conflictivas heterogéneas que tienen diferentes alcances. Las guerras civiles, por ejemplo, se produjeron como resultado, en su mayoría, de las rivalidades al interior de la clase oligarca dominante. Las temáticas imperantes en esas demandas, compartidas con otros contextos de guerra civil latinoamericanos, tuvieron que ver con el lugar de la Iglesia y sus relaciones con el Estado, la organización política de tipo centralista o federalista o la abolición de la esclavitud, por señalar algunas. La particularidad de estas guerras del siglo XIX radicó en que las rivalidades acontecieron entre grupos sociales que ocupaban un mismo status social, así pues, la clase dominante asumió tanto la dirección política como la dirección militar guerrerista (Sánchez, 2007, p. 18).

Aun con esta particularidad, las guerras se desplegaron en múltiples esferas de la vida social, estuvieron determinadas por los contextos sociopolíticos regionales o nacionales, y vinculadas con las relaciones de poder y dominio a las que contribuyeron a redefinir y configurar. De alguna manera las guerras terminaron "desplegándose hacia sectores, actividades y territorios que en principio no tenían relación con el conflicto armado, pero que terminaban articulándose con él" (Uribe, 2003, p. 29). Justamente, este despliegue tiene que ser tomado en cuenta al considerar las transformaciones de las condiciones para la manifestación conflictiva que se produjo a mediados del siglo XX, durante el periodo conocido como La Violencia. A contrapelo del periodo anterior la rivalidad no trascurrió al interior de la clase dominante, en esta oportunidad se desarrolló una crisis entre las clases dominantes y las clases subalternas que tomó fuerza durante los años treinta y cuarenta produciendo, finalmente, un estallido hacia los años cincuenta a modo de confrontación abierta. En este caso, la particularidad de la contienda estuvo dada porque aun cuando la dirección ideológica y política fue asumida por las clases dominantes, esto es, por los dirigentes de los partidos políticos tradicionales: el liberal y el conservador, la dirección en el plano militar fue llevada a cabo particularmente por el campesinado. Esta especificidad que caracteriza a La Violencia explica en buena medida sus expresiones disímiles con las guerras anteriores (Sánchez, 2007, p. 18).

Al mismo tiempo esta violencia de los años cincuenta que en un principio es partidista y que interpela a la capacidad del sistema político para ampliarse, gestó en su interior un nuevo escenario conflictivo: el insurreccional, donde los actores protagonistas, es decir, las fuerzas insurgentes campesinas, remplazaron la perspectiva de incorporación al poder por una perspectiva de sustitución del poder, interpelando así la capacidad del sistema político ya no para ampliarse, sino para transformarse (Arocha, 1995, p. 35). En este contexto nacen y se consolidan las guerrillas campesinas como las FARC (1964) y el ELN (1964) (Medina, 2010). ${ }^{2}$

Podríamos sugerir una etapa más del conflicto, aquella que inicia en la década de los ochenta caracterizada por la diversificación de los actores, la multiplicación y crecimiento de los grupos insurgentes, el auge de las Autodefensas Unidas de Colombia (AUC) conocidas coloquialmente como paramilitares y el aumento de las tensiones sociales estimuladas por la incompetencia de los gobiernos para administrar 
las fluctuaciones monetarias. Desde la perspectiva académica, en este periodo se deja de hablar de La Violencia y se empieza a hablar de las violencias, dando lugar a una explicación que desborda la dimensión política partidista y considera otras modalidades relacionándola con factores socioeconómicos, con la lucha por la posesión del territorio y señalando una violencia sociocultural por la defensa de un orden moral o social (Blair, 2009; Lamus, 2000; Ortiz, 1994; Sánchez, 1993; Urrego, 2002).

\section{Contexto histórico de La Violencia: antecedentes y desarrollo}

Hasta aquí hemos diferenciado y caracterizado las diversas etapas del conflicto colombiano teniendo en cuenta la cronología y calificación establecida por la historiografía nacional (Ortiz, 1994; Peñaranda, 2007; Sánchez, 2007). De esta manera y siguiendo el esquema presentado por el sociólogo colombiano William Ramírez (2002), a la guerra civil le dimos una calificación y un tiempo preciso de desenvolvimiento: "guerras civiles del siglo XIX", y al conflicto de mediados del siglo XX le otorgamos una presentación ortográfica particular: La Violencia (con mayúsculas), esto último para diferenciarla de los conflictos anteriores en términos cronológicos y de la violencia reciente y particular emergida a partir de los años ochenta (p. 153).

A modo de contextualización histórica, en este apartado expondremos con brevedad los antecedentes y desarrollo de La Violencia en tanto etapa particular que dividió en dos la historia del país y a partir de la cual se empezó a consolidar un campo de saberes sobre el fenómeno. Así las cosas, abordaremos tres antecedentes clave sobre los cuales ha coincidido buena parte de la historiografía colombiana, a saber: el agotamiento de la hegemonía conservadora y el establecimiento de la República liberal en el año 1930, el cambio de gobierno y retorno de los conservadores en 1946 y la muerte del candidato presidencial Jorge Eliecer Gaitán el 9 de abril de 1948.

\section{La República liberal 1930-1946}

El conflicto general de estos años surge so pretexto del cambio de gobierno: después de una larga época de hegemonía conservadora, el partido liberal obtiene el triunfo en las elecciones del año 1930 y conquista grandes posiciones políticas, inaugurando así un nuevo ciclo denominado la República liberal (1930-1946). Si bien desde el punto de vista político el Gobierno del liberal Enrique Olaya Herrera (19301934) no representó una ruptura formal con el modelo consagrado por la hegemonía conservadora -de hecho su periodo fue definido como un gobierno de concertación nacional, en el que liberales y conservadores compartieron cargos públicos y en el que las distintas tendencias intrapartidarias coexistieron-, con la llegada al poder de Alfonso López Pumarejo en 1934 la situación cambió substancialmente. A partir de este gobierno se llevó a cabo La Revolución en Marcha, un proyecto político 
donde el liberalismo adoptó parte del discurso del partido comunista y de los círculos abiertamente marxistas y a través del cual la República liberal adelantó una serie de reformas en la estructura general del país; la más importante fue la reforma constitucional de 1936, que adaptó la carta política a las exigencias económicas, políticas y sociales producidas por el régimen socialdemócrata implantado desde 1930. Dentro de sus lineamientos progresistas fue importante la reforma educativa que amplió la cobertura escolar, el reconocimiento e ingreso de las mujeres a la educación superior universitaria, el establecimiento de colegios y escuelas de carácter mixto y la propagación de la libertad de culto en las instituciones escolares.

A través de esta reforma se formalizó la estatalización de la Universidad Nacional y de las escuelas normales. Por otra parte, se legalizó la institución del sindicato y se introdujeron figuras como la función social de la propiedad, el derecho de huelga, el derecho de asociación, la intervención del Estado en la economía, el sufragio universal para los hombres, entre otros. Finalmente, el proyecto propuso una reforma agraria con el objetivo de mejorar la distribución de la tierra, la cual conllevó a la organización de la oposición conformada por diversos sectores sociales como la clase terrateniente y la Iglesia católica principalmente, para defender sus intereses frente al gobierno. Los antagonismos suscitados por las directrices de los partidos a causa del reformismo liberal tomaron forma en los sectarismos políticos de las diversas organizaciones regionales y se desenvolvieron en un enfrentamiento abierto, en zonas rurales y urbanas, de liberales contra conservadores y viceversa.

En este sentido, la historiografía colombiana registra hacia los años treinta el inicio de las confrontaciones violentas entre liberales y conservadores. De acuerdo con el historiador colombiano Miguel Ángel Urrego (2002), para esta época:

El conflicto se desarrolló buscando un cambio en la correlación de las fuerzas entre los partidos políticos a través del establecimiento de una nueva geografía de la adscripción, esto es, una reconfiguración de las zonas de hegemonía del liberalismo y del conservadurismo (p 114).

\section{El retorno del conservadurismo en 1946}

En el año 1946 el país registró un nuevo cambio político al asumir la presidencia el candidato Mariano Ospina, representante del partido conservador. La victoria conservadora se produjo bajo una gran tensión política y social reflejada en una creciente ola de huelgas y paros solidarios extendidos a lo largo y ancho del país. Para ese año el Ministerio de Trabajo reconoció más de 500 conflictos colectivos producidos por los trabajadores de las compañías de navegación del Río Magdalena, de las compañías petroleras y de las compañías de transporte. En el mes de mayo de 1947 estalló el paro general de transportes y como medida de fuerza el Gobierno conservador decidió suspender la personería jurídica a la CTC -Confederación de Trabajadores Colombianos-. Esto, por supuesto, en vez de aplacar las tensiones 
sociales las intensificó (Guzmán, Fals y Umaña, 2010, p. 43). En este marco, la movilización popular se concentró en torno a un nuevo líder político vinculado al liberalismo: Jorge Eliecer Gaitán, quien se constituyó en jefe único del partido liberal y congregó a su alrededor a una buena cantidad de cuerpo electoral. En este contexto, las mayorías liberales que se empezaron a gestar supusieron un peligro para la hegemonía conservadora, lo que derivó en la persecución política alentada por las directrices partidarias.

Durante este periodo y parafraseando al historiador Miguel Ángel Urrego (2002), la manera como se desarrolló la violencia estableció la legitimidad de la acción punitiva contra el "opositor" político y la "privatización" de la aplicación de la justicia, esto es, su imposición al margen de las instituciones del Estado. De esta forma, el choque entre campesinos liberales y conservadores no solo incluyó el castigo ejemplarizante del adversario, sino que incorporó dentro de la cultura política una vía personal para la resolución de conflictos (p. 115). Para ese entonces el país ya estaba sumido en una profunda violencia en la que se mezclaban todo tipo de divergencias que daban muestras de la extensión y magnitud del conflicto: los enfrentamientos entre bipartidistas, entre campesinos y terratenientes, entre el proletariado y el patronato, así como otros problemas (Arias, 1998, p. 40).

¡Mataron a Gaitán!: El 9 de abril de 1948

\begin{abstract}
"Mataron a Gaitán" ('ellos', los conservadores, estaba implícito en la frase) se convirtió desde entonces en el grito de batalla de aquellos liberales que tenían puestas sus esperanzas en la casi segura elección de Gaitán como presidente en 1950. Con la muerte de su caudillo veían entonces frustradas sus expectativas. (Sáenz, 2007, p. 168).
\end{abstract}

El 9 de abril de 1948 fue asesinado el "caudillo del pueblo" Jorge Eliecer Gaitán provocando verdaderas insurrecciones populares en diferentes lugares del país. En Bogotá, por ejemplo, se produjo el denominado "Bogotazo", el cual refiere a la revuelta protagonizada por una multitud enardecida que se congregó en el centro de la capital y se organizó para levantarse contra las instituciones públicas acusando al presidente y a los dirigentes del Partido Conservador de ser los autores intelectuales del asesinato del líder liberal. ${ }^{3}$

El enfrentamiento de la multitud entre sí, y de esta con la policía y el ejército durante varios días, resultó en la destrucción parcial de la ciudad y en la generalización de la violencia que se extendió hacia otras regiones urbanas y rurales del país. En un gran número de municipios, incluyendo las ciudades más grandes, se presentaron rebeliones y enfrentamientos armados: "numerosos liberales se organizaron en grupos para la acción política y armada en toda Colombia y se establecieron juntas revolucionarias para remplazar a los alcaldes y gobernadores simpatizantes 
con el régimen conservador" (Sáenz, 2007, p. 168). A partir de esta fecha insigne, La Violencia generada por el bipartidismo de las grandes ciudades se trasladó a las regiones y hacia la década de los cincuenta se estableció formalmente en los campos.

\section{La Violencia (1948-1957) y la alianza bipartidista del Frente Nacional}

Desbordando los antecedentes mencionados, la historiografía colombiana ha delimitado el periodo histórico de La Violencia específicamente entre 1948 y 1957 , para dar cuenta de la etapa conflictiva que se desata con el asesinato de Jorge Eliecer Gaitán, el 9 de abril de 1948 y que finaliza con la alianza política bipartidista del Frente Nacional en 1957. Esta etapa se caracteriza por su carácter destructivo, ya que dejó una cifra de muertos hasta hoy incalculable, una buena cantidad de desplazamientos forzados del campo a la ciudad y un potente desarraigo social.

Un episodio particular durante este periodo fue el golpe de Estado realizado en 1953 por las fuerzas militares en cabeza del general Gustavo Rojas Pinilla, cuya dictadura militar se diferenció del resto del contexto latinoamericano por ser un instrumento de las élites bipartidistas para dar salida a la crisis interna (Urrego, 2002, pp. 116-117). Este momento es catalogado por el historiador colombiano Marco Palacios (2003) como el de mayor sectarismo y fuerza destructiva del país, pues aparecieron vigorizados los denominados "bandoleros" y "pájaros": grupos de sicarios pagados por los directorios políticos que azotaron a las regiones y localidades (Jaramillo, 2011, p. 69).

La mayoría de historiadores coinciden en que este periodo de La Violencia finaliza con el derrocamiento de la dictadura militar de Rojas Pinilla en el año 1957 y con la formalización de la alianza bipartidista, política y electoral, entre liberales y conservadores, conocida como el Frente Nacional (1958-1974), cuya particularidad radica en el acuerdo de paridad entre los dos partidos, basado en la alternación en la Presidencia durante un periodo de 16 años, cuatro años cada uno: ${ }^{4}$

\footnotetext{
Los puntos de este acuerdo estipulan el establecimiento formal de un gobierno de coalición bipartidista, la distribución paritaria de los ministerios y de los cargos en el congreso, asambleas y consejos, la conformación del derecho al voto para la mujer y la legalización popular del gobierno en la Junta Militar. Para el $1^{\circ}$ de diciembre de 1957 , la Junta convocó a un plebiscito nacional para reformar la constitución, eliminando el régimen pluralista y creando el sistema bipartidista o de condominio. (Paredes y Díaz, 2007, p. 187).
}

Acontrapelo de esta delimitación, historiadores como Marco Palacios extienden la fecha de finalización de La Violencia hasta 1964, momento en que se combinan estrategias de amnistía y reinserción a la vida civil de bandas y grupos alzados en armas. Adicionalmente, es por estos años que las organizaciones guerrilleras del ELN y las FARC surgen como consecuencia de las condiciones políticas y sociales de la época $(2000$, p. 346). 


\section{LA VIOLENCIA Y LAS CIENCIAS SOCIALES}

\section{La Violencia antes de las Ciencias Sociales ${ }^{5}$}

La literatura interpretativa acerca de La Violencia -que antecede tanto al famoso libro inaugurador de este campo de estudios titulado La Violencia en Colombia y publicado en los años sesenta por Germán Guzmán, Orlando Fals Borda y Eduardo Umaña Luna, como a la consolidación formal de las Ciencias Socialesson por lo general novelas, cuentos o crónicas basados en hechos reales acontecidos en la geografía nacional, o bien, artículos de opinión en revistas y periódicos, discursos o ensayos políticos. Según el historiador colombiano Carlos Miguel Ortiz (1994), esta bibliografía, con contadas excepciones, expresa algún tipo de vínculo orgánico o afectividad con uno de los bandos en pugna: liberales, conservadores o comunistas. Sus autores son dirigentes de los partidos, funcionarios del gobierno, abogados, periodistas o combatientes de las fuerzas legales o ilegales (p. 383).

Para Ortiz (1994), este tipo de literatura que suele denominarse "partidista" comprende diferentes modalidades. Por un lado, están las obras escritas por los dirigentes políticos, liberales y conservadores, que recopilan pronunciamientos, declaraciones, conferencias y discursos en los cuales las alusiones a la violencia política son explícitas. ${ }^{6}$ Por otro lado, aparecen algunas publicaciones específicamente de denuncia basadas, por lo general, en testimonios propios o ajenos. En esta modalidad, el recurso de la novela como género literario es utilizado para dar cuenta de las torturas, masacres y asesinatos a los que eran sometidos los campesinos en las zonas rurales del país. ${ }^{7}$ Aun cuando las aproximaciones literarias fueron variadas en cuanto a temática y calidad, en su mayoría aportaron en el conocimiento social del fenómeno y contribuyeron en la denuncia del conflicto.

Específicamente, respecto a la técnica de la novela, dentro de la trayectoria literaria en Colombia y siguiendo de cerca la postura de la novelista Laura Restrepo, "la Violencia ha sido el punto de referencia obligado de casi tres decenios de narrativa: no hay autor que no pase, directa o indirectamente por el tema; éste está casi siempre presente, subyacente o explícito en cada obra" (citada por Sánchez, 2007, p. 31). ${ }^{8} \mathrm{Al}$ interior de esta modalidad, se encuentran también los libros de crónica testimonial de los combates, en estos los testimonios fueron aportados por los "rebeldes" armados o por las fuerzas militares regulares. En su momento, estos documentos se convirtieron en valiosas muestras autobiográficas que introducían al lector en las dinámicas de las guerrillas liberales particularmente, algunas con un gran sentido crítico de la coyuntura: cuestionamientos del papel de la dirigencia partidaria, de los hacendados liberales o de los jefes locales. También con sentido de autocrítica: cuestionamientos a la postura militante, a las creencias o mitos del ser "rebelde", entre otros, aportando así al entendimiento del hecho guerrillero, las luchas por el sentido y la autorepresentación: un ejemplo concreto es el libro titulado Las guerrillas del llano (1954) 
del guerrillero liberal Eduardo Franco Isaza. No obstante, la literatura testimonial de las fuerzas regulares adscritas a las instituciones estatales es mucho más desconocida y menos difundida entre el público lector, esta situación ha restringido lo que se ha dicho sobre la lógica militar interna y las relaciones de este conjunto con los grupos paramilitares. En este ámbito, el texto más divulgado fue el del coronel Gustavo Sierra Ochoa (1954) Las guerrillas en los Llanos Orientales, que desde la perspectiva del ejército documenta el nacimiento de las formas de contrainsurgencia y muestra cómo "se abre camino una estrategia más global, de combinación de la fuerza militar con la política persuasiva, lo que se llamaría más adelante «acción cívica militar»" (Ortiz, 1994, p. 386). ${ }^{9}$

Un tercer modo de literatura partidista lo conforman los libros escritos por periodistas y desde el recurso periodístico sobre fechas o acontecimientos concretos, por lo general referentes al 9 de abril de 1948 por el asesinato de Jorge Eliecer Gaitán, sobre el 13 de junio de 1953 por el golpe de Estado y la instauración del gobierno militar de Gustavo Rojas Pinilla, o acerca del derrocamiento de este régimen el 10 de mayo de 1957. La particularidad de estos escritos, que suelen argumentar desde alguna posición partidaria, está en su preocupación por fijar las responsabilidades o irresponsabilidades políticas del partido contrario o en absolver al propio. ${ }^{10}$ Finalmente, ya entrado el decenio de los sesenta fue utilizado de manera menos extensa el recurso literario del ensayo por parte de pensadores o políticos de la época. Los más significativos fueron los trabajos de Roberto Urdaneta (1960), Belisario Betancur (1961) y Mario Laserna (1961).

El historiador Gonzalo Sánchez (2007) clasifica la literatura antecedente al libro pionero de Guzmán y compañía, en apologética y testimonial. La primera es la producción elaborada, en su mayoría, durante los años cincuenta. Sobre estos escritos anota: "en ellos se revelan los elementos básicos de las representaciones que las élites o instituciones asociadas a ellas, como la Iglesia y el ejército, se hacen de la Violencia" (p. 20). Por lo general, estos documentos no tienen una intención analítica, por el contrario se caracterizan por su carácter elogioso de las dinámicas partidistas. La literatura testimonial por su parte, se caracteriza, como su nombre lo indica, por estar construida a partir de testimonios o relatos de los actores protagonistas del conflicto y dan cuenta de experiencias tanto personales como colectivas. Lo relevante de esta perspectiva es la concepción de la violencia desde los propios actores, guerrilleros, soldados o civiles partícipes del conflicto. Para este autor, si bien en el conjunto de la literatura testimonial hay un marcado acento en los aspectos militares de la contienda, también se encuentran referencias sobre las bases sociales del reclutamiento de seguidores y eventualmente sobre la vida cotidiana de las fuerzas irregulares (Sánchez, 2007, p. 22).

Apologética o testimonial, esclarecedora o denunciante, el objetivo principal de todas estas obras, según Ortiz (1994) es: 
Descubrir el responsable individual o colectivo, en el sentido de sujeto consciente productor de los actos -que son violentos y partidistas a la vez-... la tarea del que escribe se asimila aquí a la de un juez, trátese del dirigente político, del penalista que alude a su proceso, del periodista o del guerrillero que escribe sus crónicas. (p. 388).

En estos términos, el discurso con intenciones interpretativas o simplemente narrativas presupone un código ético condicionado por la coyuntura social y política, que sirve de parámetro de análisis de la realidad con el que se elabora el juicio de responsabilidades y que permite discernir, además, entre "los buenos" y "los malos". Sumado a esto, la pretensión de verdad que suponen estos escritos en su tarea de revelar a los responsables "generalmente termina siendo una justificación, incluso una apología del propio partido y una condena del partido contrario" (Ortiz, 1994, p. 388). Para Ortiz, el objetivo de la literatura sobre la violencia "partidista" demarca el principal criterio de ruptura con la producción científico-social que se desarrollará a partir de los años sesenta.

\section{La irrupción de las Ciencias Sociales en el contexto nacional}

A partir de los años treinta en Colombia y como consecuencia de los cambios económicos, sociales, culturales y políticos producidos por la incipiente urbanización, el comienzo de la industrialización y la configuración de nuevos sectores sociales, entre otros factores que favorecieron una primitiva apertura intelectual, las disciplinas denominadas "sociales" fueron buscando su carta de legitimidad para dar cuenta de la realidad, a través de procesos ambivalentes de consolidación. En este panorama, la Escuela Normal Superior, "inspirada en su homónima de París con el propósito de formar "maestros de maestros"” (Socarrás, 1987, p. 1) y fundada bajo la sombra de la Facultad de Educación en el Gobierno de la República liberal del presidente Olaya Herrera, así como la creación del Instituto Etnológico Nacional en 1941, se constituyeron en los pilares fundamentales del proceso de diferenciación de las disciplinas sociales que empezaban a abrirse camino "en momentos en que el tradicional acceso elitista a la Universidad empezaba a ceder a favor de sectores urbanos de extracción media" (Ortiz, 1994, p. 374). ${ }^{11}$

Según los datos de El Sistema Universitario en Colombia (1970), obra del historiador uruguayo Germán Rama, hasta 1940 la universidad colombiana, incluyendo tanto el sector oficial como el privado, contaba con una matrícula que rodeaba la cifra de 3000 estudiantes: "conformada por una élite que satisfacía los requerimientos de prestigio de las exclusivas clases dominantes" (Leal, 1988, p. 62). ${ }^{12}$ Luego, hacia las décadas del sesenta y setenta se produjo una profunda expansión del sistema universitario, la cual inicialmente obedeció a cambios en la organización productiva de la sociedad con la consolidación del capitalismo y la necesidad de incorporar las clases medias a la universidad, como parte de la legitimación de 
su estatus. El resultado de tal incorporación significó para el sistema un proceso de estratificación al punto de que las instituciones educativas empezaron a diferenciarse según el origen social de la población estudiantil y las diversas calidades académicas. En este contexto ciertas instituciones y facultades de orden privado, que por su prestigio social y académico absorbieron a la élite, provocaron una desvalorización social importante de los títulos académicos de los demás centros educativos, por lo general nacionales y públicos. Entre otras cosas, el deterioro del sistema universitario nacional influyó decisivamente en la creación de institutos y centros de investigación privados que, regidos por las leyes del mercado, se convirtieron en buena medida en centros de consultoría gubernamental (Leal, 1988, p. 64). Lo anterior tuvo repercusiones importantes para el naciente campo de las Ciencias Sociales, que encontró limitaciones extremas para ejercer en el ámbito de la investigación.

\section{La profesionalización de las disciplinas: el caso de la Sociología colombiana}

Antes de que las Ciencias Sociales pudieran consolidarse como tales, estas estuvieron ligadas, por un lado, a la tradición humanista de la élite donde el estudio de la lengua castellana resultaba vital en la formación humanística, y por el otro, a la carrera de Derecho, pues los abogados representaban el quehacer de la vida intelectual y la profesión "legitimaba una amplia gama de actividades de la clase dominante" (Leal, 1988, p. 64). Si bien la Escuela Normal Superior, el Instituto Etnológico Nacional y la Contraloría General de la República formaron un pequeño grupo de profesionales dedicados a la economía, la historia y la antropología (Jaramillo, 1970, p. 260), fue en la Universidad Nacional de Colombia donde se le dio fuerza a la profesionalización de las Ciencias Sociales, empezando por la carrera de Economía, la cual se consolidó en el año 1946 aunque dependiente de la Facultad de Derecho (Leal, 1988, p. 65). ${ }^{13}$ Después el turno le llegaría a la disciplina sociológica que en 1959 finalmente alcanzó su autonomía. Precisamente, en este año se fundaron tres escuelas en el país, en la Universidad Nacional, en la Pontificia Javeriana de Bogotá y en la Pontificia Bolivariana de Medellín:

\footnotetext{
Todas ellas buscaban objetivos muy similares: formar investigadores en ciencias sociales, calificar recursos humanos para realizar proyectos de cambio a nivel local, regional y nacional y desarrollar habilidades para el diseño de estrategias de planificación social. El punto central alrededor del cual giraban estos objetivos, era la necesidad de contar con especialistas que fueran capaces de enfrentar las complejidades de los fenómenos sociales en una sociedad en proceso de cambio.
}

Dentro de este panorama, los fundadores de la sociología colombiana Orlando Fals Borda y Camilo Torres jugaron un rol fundamental. Ambos, formados en el extranjero, el primero en Estados Unidos y el segundo en Bélgica, dedicaron sus energías a institucionalizar la sociología como profesión y oficio a partir de su 
conocimiento de las técnicas recientes del análisis sociológico practicado en otras latitudes. En este marco, además de las labores administrativas, ambos emprendieron investigaciones sobre la violencia, la educación, la modernización y la acción comunal, que fueron difundidas en la colección Monografías Sociológicas editada por la Facultad de la Universidad Nacional. ${ }^{14}$ Asimismo, entre los dos fundaron la Asociación Colombiana de Sociología para promover el encuentro entre sociólogos recién formados, dirigieron el VII Congreso Latinoamericano de Sociología (1964) y organizaron el I Congreso Nacional de Sociología en Bogotá (Cataño, 2008, p. 83).

Además de las Facultades de Sociología, en el año 1959 se crearon dos centros de investigación social, ambos vinculados a la Iglesia: El CINEP -Centro de Investigación Social y Educación Popular- y el Centro de Investigaciones Sociales dependiente del Arzobispado. Este contexto generó el espacio para la institucionalización de otras y novedosas disciplinas en el ámbito universitario y de esta forma, las Ciencias Sociales "rompen su cordón umbilical con la matriz jurídica... La Universidad empieza por lo menos a indagarse sobre su papel en la producción de ciencia, cultura y tecnología" (Sánchez, 1998, p. 113).

En efecto, el proyecto de institucionalización de las Ciencias Sociales estuvo atravesado por el proceso de modernización de la sociedad y del aparato productivo que revolucionó tres aspectos vitales de la sociedad colombiana de medio siglo: la educación, la secularización y el tema de las clases medias. Así las cosas, el panorama modernizador expuso grandes signos de renovación social. Por una parte, produjo una ampliación de las instituciones, productores e intermediarios culturales, así como una ampliación del público consumidor de cultura. En términos concretos, si para el año 1940 la universidad colombiana, pública y privada, contaba con alrededor de 3 000 estudiantes, para el año 1958 el número llegaba a 20000 y en 1992 superaba el medio millón (Sánchez, 1998, p. 112). Por otra parte, se produjo una ampliación de la demanda de analistas sociales y políticos.

A este respecto, como lo ha señalado el sociólogo Gonzalo Cataño (1989), Colombia siguió el patrón latinoamericano de crecimiento de las Ciencias Sociales, es decir, un desarrollo en estrecha relación con las instituciones públicas. Desde esta perspectiva, temáticas como el desarrollo agrario e industrial o movimientos campesinos y obreros, fueron los pilares del prolífero diálogo entre economistas, sociólogos e historiadores (Cataño, 1989, p. 236). Ya para finales de los cincuenta e inicios de los sesenta, sociólogos, antropólogos y geógrafos convergen en la violencia, explican, diagnostican y proponen para el Instituto Estatal Colombiano de Reforma Agraria cuya función, en ese entonces, era nuclear grupos interdisciplinarios que interpretaran la realidad conflictiva del país.

Para la historiadora colombiana Diana Obregón (1987), en este primer estadio de institucionalización de las Ciencias Sociales, los sociólogos se perciben a sí mismos como "técnicos" que pondrán su saber científico al servicio de la transformación social (Fals Borda asesora al Ministerio de Agricultura y contribuye a trazar los primeros planes de reforma agraria; Camilo Torres concibe la estrategia 
gubernamental de la "acción comunal"). En este sentido, la acción del sociólogo, según Obregón (1987), será una "acción técnica" basada en conocimiento científico, que estará al servicio de la "pura objetividad" (p. 77). No obstante, la condición de "pura objetividad" a la que alude la historiadora resulta problemática en la medida en que estos sociólogos, si bien son operativos del sistema gubernamental, también tienen un compromiso con la militancia política: Camilo Torres ingresa directamente a la guerrilla del ELN y Orlando Fals Borda promueve una diversidad de iniciativas partidistas vinculadas al segmento de la izquierda política.

\section{La Violencia en Colombia y las Ciencias Sociales}

Como vimos en apartados anteriores, la temática de la violencia fue abordada, en un principio desde la perspectiva partidista, donde los testimonios apologéticos o las responsabilidades imputadas marcaban la pauta. El enfoque propiamente científico llegaría a partir de abordajes desde disciplinas hasta entonces ajenas a las Ciencias Sociales, como el caso de la Sociedad Colombiana de Psiquiatría en cabeza de José Francisco Socarrás, quien inauguró un ciclo de conferencias titulado "Radiografía del odio en Colombia" (1959), aunque también se destacó la Comisión Investigadora de las Causas Actuales de la Violencia, encargada por la Junta Militar y creada por el Decreto 0942 de 1958, la cual es considerada como la precursora remota del libro fundador de Germán Guzmán, Fals Borda y Eduardo Umaña Luna en $1962 . .^{15}$

No obstante, ya entrada la década de los sesenta, la experiencia histórica de la violencia marcó los derroteros de análisis de las disciplinas sociales por la vía de la aproximación crítica al conflicto social y político, esto como consecuencia de las nuevas condiciones políticas generadas por los pactos bipartidistas y la reforma plebiscitaria del Frente Nacional. Siguiendo a Ortiz, efectivamente, con la repartición paritaria de los puestos públicos y el cogobierno de los dos grandes partidos tradicionales, así como los pactos de perdón y olvido celebrados por dirigentes políticos, fue perdiendo sentido la mutua inculpación de los partidos. Como resultado de lo anterior, la escritura partidista se modificó disolviendo el sujeto de inculpación en un ente bipartidista: "los dirigentes de ambos partidos fueron responsables" o en un nosotros acusado: "todos somos culpables" (Ortiz, 1994, p. 388).

En este contexto, la aparición del libro pionero La Violencia en Colombia en 1962 de Guzmán, Fals Borda y Umaña Luna supone una ruptura con la tipología literaria referida anteriormente en cuanto propone por primera vez una perspectiva conceptual desde las Ciencias Sociales, especialmente desde la Sociología. Si bien el texto no es ajeno en su totalidad a la cuestión de las responsabilidades, rebasa de lejos estos planteamientos al fijar la atención prioritariamente en la comprensión de las circunstancias que envuelven las acciones individuales y colectivas y en el funcionamiento de la sociedad. De ahí que se considere como un intento de elaboración 
"científico-social" sobre la violencia, pese a sus marcadas inconsistencias (Ortiz, 1994, p. 390).

En el abordaje del subperiodo (1948-1958) que se va a denominar La Violencia, los autores de La violencia en Colombia enmarcan el fenómeno como un proceso dentro del contexto teórico del conflicto social. De esta forma, La Violencia es interpretada como una extraordinaria acumulación de disfunciones en todas las instituciones fundamentales. Hacia las páginas finales del texto se anuncia: "existe, no obstante, una técnica dentro del proceso del conflicto que es la violencia misma, definida como el empleo de formas de coerción física para alcanzar objetos personales o de grupo" (Ortiz, 1994, p. 390). En esta medida, la primera definición conceptual adscripta al cuerpo teórico del conflicto social explica el fenómeno de la violencia, para ese periodo clásico, desde un punto de vista sociológico y científico. ${ }^{16}$

Precisamente, como lo anuncia Fals Borda en el prólogo de la primera edición, el objetivo del libro es elaborar un estudio analítico, sistemático y evaluativo de la violencia en Colombia valiéndose de una metodología de trabajo propia de las Ciencias Sociales y el empleo de una variedad de técnicas de investigación adaptadas a las circunstancias peculiares del fenómeno abordado. Es justamente la característica analítica y sistemática del estudio, aun con sus limitaciones teóricas y metodológicas, lo que convierte a este texto en el trabajo pionero y gestor de un campo de estudios sobre La Violencia, constituyendo el fenómeno en objeto de investigación y promoviendo el desarrollo de las Ciencias Sociales en Colombia (Blair, 2009, p. 24).

Cabe esclarecer en este punto otros elementos que hacen de La violencia en Colombia un hito fundador en los estudios sobre el tema. En primer lugar, se convierte en la primera aproximación académica que utiliza un enfoque interdisciplinario, pues además de la Sociología apela a las Ciencias Políticas y al Derecho. En un segundo plano, el objetivo de sistematicidad conduce a los autores a delimitar temporalmente el fenómeno (1948-1958) aunque reconociendo, desde una perspectiva histórica, la necesidad de remontarse años atrás para encontrar causas y explicaciones. En tercer lugar, se produce una configuración espacial del problema dando lugar a una primaria regionalización del fenómeno y a una consideración de la variable geográfica.

Se advierte, además, que el estudio procura una identificación de los grupos en conflicto, es decir, hay un considerable interés por reconocer a los actores protagonistas del fenómeno. En estos términos se otorga relevancia a los sectores sociales que en las usuales visiones partidistas habían sido condenados como delincuentes o culpables, o como masas fácilmente manipulables. En otras palabras, esta obra se constituye en el primer esfuerzo por globalizar el fenómeno y acceder a él por medio de una descripción detallada, construida con base en informaciones de primera mano, a partir del uso de técnicas y estrategias metodológicas propias de las Ciencias Sociales; esto es, el recorrido por las zonas afectadas, el trabajo de campo y las entrevistas orales. En conclusión y en palabras de Ortiz (1994): 
La estrategia de buscar estructuras, funciones-disfunciones, agrietamientos estructurales y 'vínculos sistémicos' antes que culpables o causas-autores, de privilegiar, en la interpretación, el papel del conflicto sobre la explicación causa-efecto unilineal, posibilita, con respecto a la bibliografía precedente, una renovación importante. (p. 391).

En efecto, además de los elementos señalados, la argumentación del libro pionero muestra interesantes realidades para las Ciencias Sociales y abre un abanico de discusiones que contribuye al desarrollo analítico del fenómeno que, más adelante, va a tener repercusiones en los marcos interpretativos sobre la violencia. ${ }^{17} \mathrm{Si}$ se sigue el esquema presentado por el historiador Gonzalo Sánchez (2007, pp. 17-32), el eje de discusiones relevantes derivadas de la mencionada obra se centra, en un primer momento, en el origen de La Violencia, el cual, al parecer de algunos analistas, tiene que ver con factores político-partidistas; dicho de otro modo, esta se origina a partir de una lucha burocrática dentro de las clases dirigentes por alcanzar la conducción del Estado. A contrapelo, una segunda hipótesis privilegia los factores socioeconómicos, esto es, apela a un carácter de clase más que de afiliación política para explicar el origen de la violencia.

Una segunda discusión se inscribe en el ámbito de los efectos particularmente económicos de La Violencia; por un lado, se advierte el fenómeno como un proceso de "refeudalización" y subdesarrollo del país y, por el otro lado, como un proceso de expropiación campesina y de expansión capitalista (Sánchez, 2007, p. 23). Una tercera discusión reconoce el impacto de La Violencia sobre las clases populares: específicamente indaga las marcas de La Violencia en la mentalidad y la conciencia política del campesinado colombiano. Desde esta perspectiva, se analiza la violencia no desde la esfera de la dominación sino desde la esfera de la rebelión, argumentando que el proceso de resistencia por parte del campesinado configura la ruptura de su tradicional actitud de sumisión, retraimiento y dispersión (Sánchez, 2007, p. 23). En contravía de esta postura aparece una perspectiva que arguye una manifiesta desconfianza por parte de la población hacia la acción de masas como instrumento de conquista de reivindicaciones campesinas. Esta discusión, luego, aportará notoriamente al campo de investigaciones referente a las luchas campesinas y a los actores del conflicto.

Como lo sugeríamos en párrafos anteriores y a partir de las discusiones desatadas por el libro La violencia en Colombia, el tema empieza a ganar estatus teórico y pertinencia como objeto de investigación en las Ciencias Sociales. De este suceso particular, dice la historiadora y socióloga Mónica Zuleta (2006):

Fue en estos años cuando las comunidades científicas convirtieron la Violencia en objeto de estudio: por un lado, seleccionaron y pusieron en relación una serie de eventos a los que asignaron ordenamientos cronológicos, espacios geográficos de manifestación y actores específicos; y por otro, les atribuyeron categorías explicativas, y lógicas organizacionales y conectivas. (p. 29). 
Al mismo tiempo, por estas décadas, pero sobre todo hacia los años ochenta, se desata una vasta producción de estudios sobre la Violencia de los cuales luego va a ser considerablemente difícil dar cuenta. Para la socióloga Elsa Blair (2009), aun con lo vasto de la producción, es posible aproximarse a los usos y significaciones de la Violencia tomando en cuenta los diversos campos disciplinares y sus objetos de estudio (p. 25). Desde esta perspectiva, la Violencia que, como vimos, es en un primer momento abordada desde la Sociología y las Ciencias Políticas, privilegia el enfoque del análisis político y por ende, el estudio de la llamada violencia política.

Coincidente con la postura de Blair (2009), Zuleta reconoce que las distintas disciplinas tienen un orden cronológico de participación en el tema. Para los años sesenta, la Violencia es problema de la Sociología, de ahí que su primaria interpretación se haga a partir de propuestas globales que intentan brindar una visión panorámica del fenómeno. Desde finales de los sesenta y durante los setenta, se vuelve objeto de la Economía y la Pedagogía; a finales de los setenta vuelve a las Ciencias Políticas y a la Antropología; por último, en los años ochenta, se aborda el fenómeno desde la Historia, la Filosofía y de nuevo la Sociología (Zuleta, 2006, p. 39).

Si nos detenemos en la producción de estudios de los años setenta y ochenta específicamente, encontramos que la Violencia es ya un eje de reflexión consolidado de las Ciencias Sociales en Colombia. Muestra de esto es la multiplicación de publicaciones, así como la realización de congresos nacionales y simposios internacionales que indican un creciente interés en el tema por parte de los productores, pero también, por parte de una audiencia receptora. ${ }^{18} \mathrm{Al}$ mismo tiempo, se renuevan las tendencias y enfoques investigativos, se reconfiguran y actualizan viejos temas de la Violencia y en relación con la coyuntura política, se ponen en primer plano problemáticas como la guerrilla, la amnistía, el proceso de paz, la rehabilitación, las reformas agraria y política, los aparatos paramilitares, entre otras.

En una perspectiva de conjunto de los estudios sobre la violencia de estos años, hay asociadas, según Sánchez (2007, pp. 17-32), tres tendencias particulares. En primer lugar, hay un tránsito del análisis de la Violencia como coyuntura política (1945-1958) a perspectivas de larga duración en las cuales el fenómeno se proyecta como un elemento estructural de la evolución política y social del país. Así, sugiere el autor, se releva un análisis de continuidades y rupturas que permite concebir las dinámicas en conjunto de las guerras civiles en Colombia. En este sentido se rescata, por un lado, el movimiento hacia atrás que empieza a considerar temas relacionados con las bases sociales de las guerras civiles, la relación entre estructuras agrarias y conflictos bélicos, las formas organizativas, la persistencia de la violencia en ciertos escenarios geográficos y en general, la permanencia de la guerra irregular en Colombia. ${ }^{19}$ Y por el otro lado, el movimiento hacia adelante, que tiene que ver con el impacto de la situación política contemporánea para los años ochenta.

En segundo lugar, hay una tendencia que tiene que ver con el desplazamiento de estudios globalizantes a estudios regionales, a unidades temáticas o a coyunturas específicas. En esta tendencia, los estudios regionales han tratado de definir 
las relaciones entre estructura agraria, estructura de clase y conflictos sociales; las unidades temáticas se aproximan particularmente al fenómeno del bandolerismo y su relación con las organizaciones campesinas o la relación clase obrera/violencia, iglesia/violencia o ejército/violencia; mientras los estudios de coyuntura han centralizado su atención en los acontecimientos del 9 de abril de 1948, demostrando que los incidentes no solo se desarrollaron en Bogotá, sino que tuvieron lugar en las zonas rurales más apartadas del centro político del país.

En tercer lugar, la última tendencia perceptible en el conjunto de estudios renovados sobre la violencia, es el gradual desprendimiento de un cierto economicismo dominante en la producción de los años setenta, que enmarca el tema dentro de los parámetros de la dupla violencia/capitalismo; en su lugar, comienzan a ganar espacio las determinaciones políticas, sociales y culturales del fenómeno.

Así las cosas, de la mano de la proliferación de estudios sobre el tema hay una reinterpretación del fenómeno que da lugar a nuevas significaciones históricas de los sucesos acontecidos durante el periodo de la denominada Violencia. En esta medida, el panorama general sobre el fenómeno cambia, así como las maneras de abordarlo desde las Ciencias Sociales. Precisamente con la expansión de estas disciplinas y la introducción de una concepción orgánica de la sociedad que trabaja con la noción de Estado como instancia encargada de ejercer el monopolio sobre el ejercicio de la violencia, se empieza a pensar el fenómeno, principalmente, en términos de fallas en la estructura estatal (Rueda, 2008, p. 353).

\section{Las Ciencias Sociales en el panorama latinoamericano}

Aunque este artículo se centra en el proceso de institucionalización de las Ciencias Sociales en el contexto colombiano, es preciso dar cuenta del panorama general en el ámbito latinoamericano, para distinguir así las diversas rutas que se adoptaron en el camino. Ya que dar cuenta del proceso de la región es un trabajo en sí mismo y excede los propósitos del presente artículo, se toma como referencia comparativa del caso colombiano, aunque de manera breve y superficial, los casos de Argentina y México.

De acuerdo con José Luis Reyna (2004), puede afirmarse que la institucionalización de las Ciencias Sociales en América Latina se origina, aproximadamente, durante la segunda mitad del siglo XX (p. 490), aunque con procesos diferenciados según la particularidad de cada país. En efecto, el devenir histórico, político y social condicionó el desarrollo de las Ciencias Sociales en cada contexto.

Para el caso argentino, por ejemplo, y siguiendo la postura del sociólogo Carlos Altamirano (2004), al igual que en el conjunto de América Latina, la institucionalización y profesionalización de las Ciencias Sociales no fueron resultado de una reflexión originada al interno de la comunidad académica local, sino adoptada de reflexiones externas y adaptada a la vida social de las comunidades criollas. En este 
marco, una incipiente institucionalización se vislumbra hacia finales del siglo XIX e inicios del XX, donde espacios como la Facultad de Derecho y Ciencias Sociales y la Facultad de Medicina de la Universidad de Buenos Aires se constituyeron en los centros autorizados para hablar en voz alta sobre las cuestiones sociales (Altamirano, 2004 , p. 33). Sus miembros, quienes poseían capital cultural y un conjunto de saberes específicos, se fueron integrando a la institución universitaria, la cual se desarrollaba al mismo tiempo que avanzaba el proceso de modernización económica y social. En este contexto, por ejemplo, la Sociología aparece en 1886 como asignatura y con el nombre de Ciencia Social dentro del plan de estudios de la Facultad de Filosofía y Letras, no obstante, la cátedra no se regulariza hasta 1904 (Altamirano, 2004, p. 38).

Autores como Guber, Gurevich y Visacoksky (1997), sin embargo, sugieren que el punto cumbre de institucionalización de las Ciencias Sociales en Argentina, se encuentra en los proyectos modernizadores de los años cincuenta que, vehiculizados desde el Estado, se plasmaron en el campo académico-intelectual. Así pues, en 1957 las cátedras como Sociología, Psicología y Ciencias de la Educación pasan a ser carreras, convirtiéndose así en la expresión del proyecto político nacional posperonista (1997, p. 214).

Dicho lo anterior, la institucionalización de las Ciencias Sociales en Argentina estuvo fuertemente ligada al contexto político de la época que buscó, a través del favor de las Ciencias Sociales, legitimar el derrocamiento del presidente Juan Domingo Perón en 1955 y “desperonizar” a la población. De esta manera, a partir de 1955 y con el propósito de contribuir a la modernización y el desarrollo social de la nación, se reestructuró la universidad, reformándola sustancialmente a nivel institucional. En este marco, figuras como el sociólogo Gino Germani, el historiador Luis Romero y el psicólogo Enrique Butelman, fueron fundamentales.

No obstante, después de su institucionalización y arraigo en las universidades a lo largo y ancho del país, y como consecuencia de las fluctuaciones a nivel social y político de la época, particularmente por los golpes de Estado y las dictaduras militares, "en los países del Cono Sur las ciencias sociales fueron prácticamente expulsadas de las universidades, con consecuencias que aún después del retorno a la democracia, sería difícil sobreestimar" (Lander, 1997, p. 21). En este marco, las disciplinas sociales tuvieron que desplazarse hacia los centros privados y buscar financiamiento externo para desarrollar proyectos de investigación.

El caso argentino difiere en buena medida del proceso de institucionalización de las Ciencias Sociales en el contexto mexicano, comenzando por el punto de partida. En México, por los antecedentes prehispánicos culturales y el desarrollo de las civilizaciones indígenas y su riqueza arquitectónica, disciplinas como la Historia y la Antropología se abrieron camino asumiendo una perspectiva más institucional (Reyna, 2004, p. 65). De hecho, son estas las que le darían paso a la Sociología y las Ciencias Políticas por ejemplo, en contraposición a los casos de Argentina y Colombia.

De acuerdo con Reyna (2004), después del movimiento revolucionario de 1910 y frente a un contexto de estabilidad política arranca la fundación de instituciones 
académicas que, financiadas por el Estado, contribuyeron al desarrollo de las disciplinas sociales. En este punto, la particularidad del caso mexicano también se diferencia de los casos de Argentina y Colombia, donde las relaciones con el Estado han sido variadas: en algunos momentos cercanas, en otras oportunidades distantes. De hecho, la institucionalización de las Ciencias Sociales para el caso argentino tuvo como estímulo particular los recursos económicos provenientes del exterior. Sobre este punto, las discusiones que abordan la temática del papel crítico de los intelectuales frente a los regímenes de poder tienen gran importancia.

De acuerdo con lo dicho hasta el momento, podemos advertir dos cosas: por un lado, que las disciplinas varían en cuanto a su importancia. Así pues, en el caso argentino y colombiano, la Sociología se consolidó prontamente debido a su alta demanda en contraste con la baja demanda de las otras disciplinas. En México, no obstante, la Antropología y la Historia se afianzaron dándole posibilidad de emerger a otras áreas de conocimiento. Por otro lado, advertimos que el enfoque de los problemas, los objetos de estudio y la orientación teórica y metodológica también varían dependiendo de las condiciones sociales, políticas e históricas de cada país. Si en Colombia el tema de la violencia se convirtió en el hilo transversal de discusión y trabajo, en México, por ejemplo, ese hilo lo constituyó la discusión sobre la herencia cultural prehispánica, mientras que en Argentina la discusión estuvo mediada por la pretensión de "desperonizar a las masas", y evaluar las causas y consecuencias de la inestabilidad política reflejada en los sucesivos golpes de Estado.

Sin embargo, así como los puntos de partida para la institucionalización difieren y las temáticas se diversifican, hay líneas en común que atraviesan los tres casos y que, en general, podrían extenderse al contexto latinoamericano. En definitiva, la institucionalización permitió que las disciplinas sociales encontraran su lugar particular en el amplio campo del conocimiento académico e intelectual, dando lugar a que tuvieran sus propias instituciones, sus propios objetos de estudio, sus propios intereses temáticos y sus propias metodologías. De igual manera, la institucionalización posibilitó, además, el vínculo con la universidad latinoamericana y con el mercado de trabajo, estableciendo así la profesionalización de las disciplinas (Reyna, 2004, p. 492).

\section{CONCLUSIONES}

La institucionalización de las Ciencias Sociales en Colombia, en cuanto corpus de conocimientos, problemas y técnicas de investigación, procesados sistemáticamente y reconocidos y aprobados por una comunidad epistémica, repercutió sustancialmente en la construcción de un campo de saber dedicado especialmente al tema de la violencia. En este marco, no fueron pocos los efectos producidos por la aparición de la obra de Guzmán y compañía, pues en definitiva, esta señaló la necesidad de una modalidad responsable de realizar la tarea investigativa, 
lo cual implicaba dejar de lado cualquier toma de posición partidaria y oponerse al modo de conocimiento práctico que se hallaba en la experiencia ordinaria de la violencia. Esto significó la puesta en cuestión de la literatura testimonial, la crónica periodística y el recurso de la novela, en razón de su escasa fiabilidad al no contar con el carácter "objetivo" de la investigación social. En esta medida, la nueva manera de concebir la violencia -como objeto de estudio- y la actividad intelectual, se impuso a toda una generación y la investigación académica empezó a ocupar una posición dominante en el campo de producción de saber sobre la violencia.

Finalmente, este trabajo resulta de vital importancia en la medida en que contribuye al campo de conocimiento más general que se encarga de indagar la reconstrucción histórica de las Ciencias Sociales en América Latina. Es decir, se suma al acervo de estudios, a nivel regional, que se han encargado de examinar la constitución e historia de las Ciencias Sociales. En este marco, el interés particular por el caso colombiano contrasta con los estudios que se han ocupado reiteradamente de las experiencias nacionales de México, Argentina y Brasil; aunque en este punto vale la pena mencionar que este panorama ha empezado a cambiar recientemente y han aparecido valiosas investigaciones de países latinoamericanos más pequeños y aportes empíricos novedosos.

En definitiva, este trabajo pretende contribuir al debate sobre la historia de las Ciencias Sociales en el marco regional latinoamericano desde la perspectiva local colombiana, considerando el impacto de las tradiciones intelectuales que pensaron y definieron la violencia, y construyeron redes académicas y sociales que legitimaron esos saberes producidos en el mismo seno de las Ciencias Sociales.

\section{NOTAS}

1 Gonzalo Sánchez, filósofo, abogado, historiador y sociólogo, es acaso la figura más prestigiosa entre los académicos estudiosos de la violencia. Desde una perspectiva historiográfica, Sánchez entiende el conflicto colombiano a partir de una lectura de largo plazo. De esta forma, opina, existen regularidades explicativas sobre la violencia que dejan una huella persistente en nuestra memoria nacional, especialmente sobre la memoria de una guerra que no es coyuntural, sino de largo alcance y que se remonta hasta finales del siglo XIX (Jaramillo, 2011, p. 212). Esta lectura sería clave para consolidar en el imaginario nacional la idea de la existencia de una tendencia identificable históricamente respecto de la violencia colombiana. En el presente trabajo, Sánchez es citado recurrentemente, pues su estudio de la violencia a largo plazo permite contextualizar el fenómeno para el público que no está familiarizado con el conflicto colombiano. De la misma manera, Sánchez es un estudioso del campo de estudios sobre la violencia y sus contribuciones en este ámbito resultan de vital importancia.

2 Para profundizar sobre la historia de las FARC y el ELN, remitirse a (Medina, 2010).

3 En la crónica sobre el 9 de abril hecha por el historiador Herbert Braun (2007), este relata: "La multitud estaba formada por varias clases sociales, incluía las capas medias, los profesionales, empleados estatales, municipales, electricistas..., prostitutas y criminales. Mujeres jóvenes y 
viejas que tenían sus puestos en los mercados, se unieron a las multitudes en grupos, agitando banderas y guiando el ataque de los edificios" (p. 223).

Los presidentes durante el periodo del Frente Nacional fueron: Alberto Lleras Camargo (liberal) 1958-1962; Guillermo León Valencia (conservador) 1962-1966; Carlos Lleras Restrepo (liberal) 1966-1970; Misael Pastrana Borrero (conservador) 1970-1974.

Tomando como referencia el texto del historiador colombiano Marco Palacios (1994) Modernidad, modernizaciones y ciencias sociales, en este trabajo entendemos el término ciencia social como un corpus de conocimientos, problemas y técnicas de investigación, todos ellos procesados sistemáticamente y reconocidos y aprobados por una comunidad epistémica.

La publicación más representativa de esta modalidad es la antología de discursos e intervenciones del presidente liberal Carlos Lleras Restrepo (1955), titulada De la República a la Dictadura. Testimonio sobre la política colombiana. (Colombia: Editorial Argra). Por parte del partido conservador, el primer secretario del presidente Ospina Pérez, Rafael Azula Barrera (1956), escribe un libro cuyo título parece interpelar al de Lleras: De la revolución al orden nuevo: proceso y drama de un pueblo.

Dentro del corpus literario novelístico que refiere la violencia partidista sobresalen autores como Fidel Blandón Berrío (1955) Lo que el Cielo no perdona; Daniel Caicedo (1954) Viento Seco; Clemente Airo (1961) La ciudad y el viento; Tulio Bayer (1960) Carretera al mar; Carlos Esguerra Flórez (1954) Los Cuervos tienen hambre; Euclides Jaramillo Arango (1959) Un campesino sin regreso; Hipólito Jerez (1955) Monjas y bandoleros. Estas novelas, que hacen parte de un acervo mucho más grande, se caracterizan por su interés testimonial y descriptivo, a contrapelo de otros títulos que, gestados en la misma época, tienen un carácter más literario y artístico, tales como La Mala Hora (1962) de García Márquez, Siervo sin Tierra (1954) o El Cristo de Espaldas (1952) de Eduardo Caballero Calderón, Cenizas para el viento (1950) de Hernando Téllez, o El Gran Burundú-Burundá ha muerto (1966) de Jorge Zalamea.

Para ampliar el tema, véase: Suárez, G. (1966). La novela sobre la violencia en Colombia; Álvarez, G. (1970). La novelística de la violencia en Colombia; Restrepo, L. (1986). Niveles de realidad en la literatura de la 'violencia' colombiana; Mena, L. (1978). Bibliografía anotada sobre el ciclo de la violencia en la literatura colombiana; Arango, M. (1985). Gabriel García Márquez y la novela de la violencia en Colombia.

"La estrategia contrainsurgente del Estado estuvo centrada en aislar políticamente a la guerrilla de la población civil... en las áreas críticas de la época. Desde el punto de vista denominado "orden público" se caracterizó por la inauguración de las "contraguerrillas profesionales", por la acción cívico militar y la acción psicológica sobre la población civil" (Torres y Rodríguez, 2008, p. 325). El plan de Acción Cívica Militar denominado en Colombia Plan Lazo es, para algunos analistas, "una estrategia contrainsurgente de tipo preventivo implementado por el Ejército con la asesoría y coordinación de los Estados Unidos" (Torres y Rodríguez, 2008, p. 326). Para profundizar en este punto, véase entre otros: Gallón, G. (1983). La república de las armas; Posada, M. (1975). Ejército y poder burgués en Colombia: El periodo del Frente Nacional. Estampas y testimonio de la Violencia. O los de Abella, A. (1973). Así fue el 9 de abril; (1973). Asífue el 13 de junio. (1938-1942) y sus actividades investigativas fueron criminalizadas durante los años cincuenta 
por las posturas políticas de su director Paul Rivet, "quien sostenía tesis que ofendían el credo nazi-fascista europeo y el de sus epígonos colombianos" (Sánchez, 1998, p. 110).

Según la filósofa y educadora Diana Soto (2005), "los estudiantes en la educación superior oficial, hacia 1935, casi triplicaban a los de la privada y en total se localizaban 3.687... La relación se mantiene hasta 1945 cuando se da un aumento considerable en la matrícula de estudiantes con 6.512 y de estos, 4.730 en la oficial" (p. 124).

"La Escuela Normal albergaba a la mayor parte de las grandes figuras de las ciencias sociales contemporáneas en el país. Sólo unos pocos, igualmente influyentes, formados en otras escuelas y en otras latitudes, quedaban por fuera de esta lujosa nómina: los filósofos Rafael Carrillo y Danilo Cruz Vélez de la recién creada (1945) Facultad de Filosofía y Letras de la Universidad Nacional; los historiadores Guillermo Hernández Rodríguez y Luis Ospina Vásquez; el arqueólogo Gonzalo Correal, y el economista Antonio García, entre los colombianos; entre los extranjeros, que vinieron por otro camino a quedarse, figuraban, por último, Juan Friede y Gerardo Reichel-Dolmatoff”' (Sánchez, 1998,p. 110).

14 Orlando Fals Borda inicia su carrera académica en el año 1953 con la publicación de artículos en revistas académicas y de corte cultural. En 1955 es publicada, en Estados Unidos, su tesis doctoral Peasant society in the Colombian Andes: a sociological study of the Saucio, traducida al español en 1961 como: Campesinos de los Andes: estudio sociológico de Saucío, la cual lo ubicaría como un especialista del desarrollo y las perspectivas de la sociología rural en Colombia y América Latina. Luego, sería reconocido por introducir en las Ciencias Sociales colombianas la técnica metodológica denominada Investigación Acción Participativa. Por su parte, Camilo Torres, sacerdote ligado a la corriente de la Teología de la Liberación, inició su carrera académica con la elaboración de la tesis doctoral en la Universidad de Lovaina titulada: Una aproximación estadística a la realidad socioeconómica de Bogotá, obra pionera en sociología urbana en Colombia que después fue publicada en 1987 con el título de La proletarización de Bogotá. En 1963, publica La violencia y los cambios socioculturales en las áreas rurales colombianas y en 1964, La desintegración social en Colombia está gestando dos subculturas. En 1965, ingresa a la organización guerrillera ELN y en 1966 muere en su primer combate contra el Ejército Nacional.

15 La Comisión estuvo integrada por dos representantes de los partidos políticos tradicionales, dos militares y dos sacerdotes. Según el artículo 5 del Decreto 0942, citado por Germán Guzmán en Reflexión Crítica sobre el libro La Violencia en Colombia (2007): "La comisión tendrá la autoridad necesaria para conducir su acción investigadora a todos los sitios que juzgue convenientes, tener acceso a todas las dependencias oficiales y enterarse de todos los informes oficiales, de carácter público, reservado o secreto, así como de los sumarios y demás expedientes que se adelanten, con el solo fin de basar sus opiniones en hechos concretos" (p. 47).

16 "Los maestros que inspiran la conceptualización - ellos lo dicen expresamente- son los 'estructural funcionalistas': los clásicos Parsons y Merton, Lewis Coser con su teoría del conflicto y Charles Loomis con su propuesta de sistemas y relaciones intrasistémicas e intersistémicas. El capítulo XIII del tomo I, en particular, es una síntesis del corpus teórico que se han propuesto utilizar para desentrañar los fenómenos estudiados" (Ortiz, 1994, p. 391).

17 "Después de Guzmán, en los doce años siguientes, es poco lo que la sociología colombiana aporta a la comprensión de la violencia... El vacío lo llenan entonces los politólogos norteamericanos, iniciadores de una discusión que llevará, en la segunda mitad de los años 70, a colocar el problema de la violencia en el centro de las preocupaciones de la ciencia política (nacional y extranjera sobre Colombia), desplazando casi por completo las otras temáticas" (Ortiz, 1994, p. 392). 
18 Dentro de las actividades académicas vinculantes sobre la violencia en la década del ochenta se encuentra: El Congreso Nacional Sobre la Violencia en Colombia. Abril de 1982 en Bogotá; junio de 1982 en Chiquinquirá; y el Primer Simposio Internacional sobre la Violencia en Colombia, que se llevó a cabo del 24 al 30 de junio de 1984 en Bogotá, patrocinado por el Departamento de Historia de la Universidad Nacional de Colombia y el Centro Cultural Jorge Eliecer Gaitán.

19 Desde la perspectiva de este autor "Colombia ha sido un país de guerra endémica, permanente" (2007, p. 17).

\section{REFERENCIAS}

Altamirano, C. (2004) Entre el naturalismo y la psicología: el comienzo de la "ciencia social" en la Argentina. En Neiburg, F. y Plotkin, M. (Comps), Intelectuales y Expertos. La constitución del conocimiento social en la Argentina (pp. 31-65). Buenos Aires: Paidós.

Arias, R. (1998). Los sucesos del 9 de abril de 1948 como legitimadores de la violencia oficial. Historia Crítica, (17), 39-46.

Arocha, J. (Coord.). (1995). Colombia: Violencia y Democracia. Colombia: Comisión de Estudios sobre la Violencia.

Blair, E. (2009). Aproximación teórica al concepto de violencia: avatares de una definición. Política y Cultura, (32), 9-33.

Braun, H. (2007). Los mundos del 9 de Abril, o la historia vista desde la culata. En G. Sánchez y R. Peñaranda (Comps.), Pasado y Presente de la Violencia en Colombia (pp. 199-228). Colombia: La Carreta Editores.

Cataño, G. (1989). Educación y Estructura Social: Ensayos de sociología de la educación. Plaza \& Janés. Asociación Colombiana de Sociología.

Cataño, G. (2008). Orlando Fals Borda: Sociólogo del compromiso. Revista de Economía Institucional, 10(19), 79-98.

Chernick, M. (octubre/diciembre, 1996). Aprender del pasado: breve historia de los procesos de paz en Colombia (1982-1996). Revista Colombia Internacional, (36), 4-8.

Guzmán, G., Fals, O. y Umaña, L. (2010). La violencia en Colombia. (Tomos I y II). Colombia: Alfaguara.

Jaramillo Uribe, J. (1970) Notas para la historia de la Sociología en Colombia. En Bateman, A. Apuntes para la historia de la ciencia en Colombia (pp.239-261) Bogotá: Colciencias.

Jaramillo, J. (2011). Las comisiones de estudio sobre la violencia en Colombia: tramas narrativas y ofertas de sentido temporal para comprender la violencia. (Tesis de Doctorado en Ciencias Sociales). Facultad Latinoamericana de Ciencias Sociales, México. 
Lamus Canavate D. (junio, 2000). Investigación social y violencia en Colombia. Reflexión Política, $2(3)$, .

Lander, E. (julio/agosto, 1997). Las ciencias sociales en el atolladero. América Latina en tiempos posmodernos. Revista Nueva Sociedad, (150), 19-23.

Leal, F. (1988). La profesionalización de los estudios políticos en Colombia. Análisis Político, (3), 61-79.

Medina, C. (2010). FARC-EP y ELN. Una historia política comparada 1958-2006. (Tesis Doctoral en Historia). Universidad Nacional de Colombia, Colombia.

Obregón Torres, D. (1987). Sociología: de la palabra al concepto. Una hipótesis sobre la constitución de la sociología como ciencia en Colombia. Revista Colombiana de Sociología. (5), 71-78.

Ortiz, C. (1994). Historiografía de la Violencia. En B. Tovar (Comp.), La historia al final del milenio (pp. 371-423). Colombia: Universidad Nacional de Colombia.

Palacios, M. (1994). Modernidad, modernizaciones y ciencias sociales. Análisis Político, (23), 5-33.

Palacios, M. (2000). La solución política al conflicto armado, 1982-1997. En A. Camacho y F. Leal (Comps.), Armar la paz es desarmar la Guerra (pp. 345-401). Colombia: Instituto de Estudios Políticos y Relaciones Internacionales.

Palacios, M. (2003). Entre la legitimidad y la violencia en Colombia 1875-1994. Colombia: Editorial Norma.

Paredes, Z. y Díaz, N. (enero/junio, 2007). Los orígenes del Frente Nacional en Colombia. Revista Presente y Pasado. Revista de Historia, (23), 179-190.

Peñaranda, R. (2007). La guerra en papel. Balance de la producción sobre la violencia en los años noventa. En G. Sánchez y R. Peñaranda (Comps.), Pasado y Presente de la Violencia en Colombia (pp. 33-46). Colombia: La Carreta Editores.

Pizarro, E. (2015). Una lectura múltiple y pluralista de la historia. Recuperado de: http://www.altocomisionadoparalapaz.gov.co/oacp/Documents/relatoria-eduardo-pizarro-leongomez.pdf/

Rama, G. (1970). El Sistema Universitario en Colombia. Colombia: Universidad Nacional de Colombia.

Ramírez, W. (2002). ¿Guerra civil en Colombia? Análisis Político, (46), 151-163.

Reyna, J. L. (mayo/agosto, 2004). La institucionalización y profesionalización de las Ciencias Sociales en América Latina. Estudios sociológicos, 22(2), 483-493.

Rueda, M. (2008). Nación y narración de la violencia en Colombia. De la historia a la sociología. Revista Iberoamericana, 84(223). 345-359. 
Sáenz, E. (2007). La ofensiva empresarial: industriales, políticos y violencia en los años 40 en Colombia. Colombia: Colección CES, Universidad Nacional de Colombia.

Sánchez, G. (1993). Los intelectuales y la violencia. Análisis Político, (19), 40-50.

Sánchez, G. (1998). Intelectuales, poder y cultura nacional. Análisis Político, (34), 99-119.

Sánchez, G. (2007). Los estudios sobre la violencia: balance y perspectivas. En G. Sánchez y R. Peñaranda (Comps.), Pasado y Presente de la Violencia en Colombia (pp. 17-32). Colombia: La Carreta Editores.

Socarrás, J. (1987). Facultades de educación y Escuela Normal Superior: Su historia y aporte científico, humanístico y educativo. Colombia: Ediciones La Rana y el Águila.

Soto, D. (2005) Aproximación histórica a la universidad colombiana. Revista Historia de la Educación Latinoamericana, 7, 101-138.

Torres, C. y Rodríguez, S. (2008). De milicias reales a militares contrainsurgentes. La institución militar en Colombia del siglo XVIII al XXI. Colombia: Editorial Pontificia Universidad Javeriana.

Uribe, M. (2003). Las guerras civiles y la negociación política: Colombia, primera mitad del siglo XIX. Revista de Estudios Sociales, (16), 29-41.

Urrego, M. A. (2002). Intelectuales, estado y nación en Colombia: de la guerra de los mil días a la constitución de 1991. Colombia: Siglo del Hombre Editores.

Visacovsky, S., Guber, R. y Gurevich, E. (octubre, 1997). Modernidad y tradición en el origen de la carrera de Ciencias Antropológicas de la Universidad de Buenos Aires. Revista Redes, 4(10), 213-257.

Zuleta, M. (octubre, 2006) La violencia en Colombia. Avatares de la construcción de un objeto de estudio. Revista Nómadas. (25) 54-69. 